\title{
FLOWS IN MAGNETIC FLUX TUBES
}

\author{
C.J. Durrant \\ Fraunhofer-Institut, Freiburg
}

Harvey has reported in this discussion observations of mean downflows in magnetic elements that increase in speed with depth. It is therefore pertinent to ask what role such flows may play in the production and maintainance of the flux concentrations.

\section{EQUILIBRIUM MODELS}

The first thing to be said is that such flows cannot directly influence the horizontal momentum balance, i.e. their dynamic pressure is negligible.

A steady internal flow such as that proposed by Ribes and Unno (1975) for chromospheric concentration would require supersonic flows with very large curvature at photospheric levels. There is no evidence for transverse flows of such magnitude.

The rejection of such possibilities implies the rejection of the mechanism proposed by stenflo (1973) inspired by the general argument of Sreenivasan (1973). This envisages the production of a magnetic field with energy density greater than the equipartition value by evolving a force-free field as some sort of 'response' to a particular velocity field, a so-called hydromagnetic Beltrami flow, that maintains the force-free character of the field. However, by definition, the local velocity field can have nothing to do with the increase of magnetic energy as the concentration occurs. The crucial point to note is that, in an isolated fluid mass, the only field that is force-free everywhere is the trivial case in which it is identically zero. Any increase in magnetic energy in a force-free region is due entirely to the work done at the boundary and the energy transport across the boundary in response to work done in the external, non-forcefree, region.

In the absence of dissipation, the rate of change of magnetic 
energy $M$ in a force-free region $V$, bounded by the surface $S$ is

$$
\begin{aligned}
& \frac{\partial M}{\partial t}=\frac{\mu}{4 \pi} \int_{V} \underline{v} \times \underline{H} \cdot \nabla \times \underline{H} d V-\underline{\mu} \int_{4 \pi} \underline{H} \times(\underline{V} \times \underline{H}) \cdot \underline{d S} \\
& =-\frac{\mu}{\Delta \pi} \int_{S} H^{2} \underline{v} \cdot \underline{d S}+\underline{\mu} \int_{\Delta \pi}(\underline{H} \cdot \underline{v}) \underline{H} \cdot \underline{d S} \\
& \text { flux of magnetic rate of working } \\
& \text { energy across boundary at boundary }
\end{aligned}
$$

Concentration arises through changing surface stresses. Naturally there exist pathological internal flow fields that maintain the force-free nature of the field as it evolves. Perhaps the simplest example is that of a squeezed uniform field

$$
\begin{aligned}
& \underline{\mathrm{B}}=\left(0,0, \mathrm{v}_{0} \mathrm{~B}_{0} \mathrm{t}\right) \\
& \underline{\mathrm{v}}=\left(\mathrm{v}_{\mathrm{o}} \mathrm{x}, 0,0\right)
\end{aligned}
$$

The magnetic energy and flux within fixed boundaries at $x= \pm X$ continually increase but the configuration remains force-free within the boundaries. Work is done in pushing the field across the boundary.

However, in general, the internal flow is governed by an energy equation not by the maintainance of a force-free state. If the magnetic energy density is greater than the flow and thermal energy densities, any magnetic stresses will tend to be transferred by Alfven waves to the boundaries and an almost-force-free state will arise. On the other hand, there is no guarantee that the flow will even approach the Beltrami-type since relatively small departures from a force-free magnetic field configuration will cause large changes of pressure.

Thus we conclude the only sufficiently general and uniform force field that could maintain a magnetic concentration is the external thermal gas pressure. This allows balancing with

$$
\frac{\mathrm{B}^{2}}{8 \pi} \sim \mathrm{p}_{\mathrm{go}} \lesssim \mathrm{p}_{\mathrm{gi}}
$$

This reduced internal pressure further implies that in the observable layers the field must be almost force-free except at the boundary of the tube. The field lines of such a thin flux tube will be almost straight in the low photosphere.

However, flows cannot be ruled out if the density in the tube is small enough and the tube narrow enough for mass balance in the 
atmosphere to be no problem. In this case the stratification need not even be close to hydrostatic along the field lines.

It may be argued that pores and sunspots show no great departures from these conditions so that in the absence of physical inconsistency we should first examine the possibilities of the simplest static model, an approach which has been successfully pursued by spruit and described in this discussion.

\section{STABILITY OF MODELS}

Flows might not be necessary in order to construct equilibrium models but there is an implicit suggestion that they might be necessary for stability. For example, linear stability analysis shows that an infinite, twisted tube embedded in a conducting, incompressible medium is unstable in the absence of flow, but that it can be stabilized if the flow energy exceeds the magnetic energy (Chandrasekhar, 1961).

The equipartition solution in which flow along field lines has an energy density equal to the magnetic energy density is always stable. Parker (1976a) has shown that this case has the interesting property that a slow systematic variation of the pressure around a particular thin flux tube causes the internal conditions to evolve close to successive equipartition solutions. Pressure changes communicated to the tube interior can cause the flow to accelerate, the field to increase and the tube to shrink (in order to conserve flux). Presumably any final concentration thus produced is stable.

No such simple solution is possible in a stratified medium. The demands of both flux and mass conservation are incompatible with it. Instead, Parker $(1976 \mathrm{~b})$ shows that in a one-dimensional approximation to a very thin flux tube, only one type of steady flow can exist that stretches from the top to the bottom of the atmosphere. In this flow, a neck occurs at the point where the scale height gradient is equal to $-1 / 2$ and only there are the flow and magnetic energies equipartitioned. In the solar atmosphere this occurs at the top of the convection zone. But this result seems of doubtful relevance to the present discussion for the following reasons:

1. Existence theorems based on first-order terms of expansions should be regarded with suspicion especially when such terms cannot be small everywhere.

2. Parker does not demonstrate that the configuration is stable nor that it can evolve naturally from mild to strong concentration.

3. The process breaks down when the flows approach the sound speed and compressibility effects must be taken into account. This 
limits the degree of pressure reduction since

$$
\frac{B^{2}}{8 \pi} \sim \frac{1}{2} \rho v^{2}<\frac{1}{2} \rho c_{s}^{2} \simeq P_{g i} \text { and } \frac{B^{2}}{8 \pi}+P_{g i} \simeq p_{g \circ}
$$

and hence

$$
P_{g i}>\frac{1}{2} P_{g \circ}, \quad \frac{B^{2}}{8 \pi}<\frac{1}{2} P_{g \circ}
$$

whereas observations imply a greater reduction which can be achieved by limiting the energy input. The less efficient mechanism is unlikely to stabilize the more efficient one.

These dynamic considerations are only essential so long as one accepts the premise that simple hydrostatic solutions are unstable. The recent analyses of Meyer et al. (1977) and Wilson, reported here, show that axial cooling of sunspots and, by extension, smaller flux tubes, is sufficient to guarantee stability. There is then no compelling theoretical requirement for steady flows in flux tubes.

On the other hand, it might be argued that strong, steady flows are unlikely. If material flows out of both ends of a tube, either into subsurface regions or into the corona, the tube would be rapidly evacuated and then what? Giovanelli (1976) has suggested that the ionization in the temperature minimum regions is low enough to allow a leakage of neutral material across the lines of force and into the flux tube, but it is doubtful whether such a mechanism can supply flows of the quoted speeds.

We must therefore conclude that our present understanding of the physics of magnetic flux elements does not rely on the presence of downdrafts within the elements. They should be regarded as a transient detail of our model and not an intrinsic feature.

References

Chandrasekhar, S.: 1961, Hydrodynamic and Hydromagnetic Stability, 0xford Univ. Press, Oxford, p. 551 ff.

Giovanelli, R.G.: 1976, Private communication

Meyer, F., Schmidt, H.U., Weiss, N.0.: 1977, submitted to Monthly Notices Roy. Astron. Soc.

Parker, E.N.: 1976a, in preparation

Parker, E.N.: 1976b, in preparation

Ribes, E., and Unno, W.: 1975, Proc. First European Solar Meeting, Feb. 1975, Firenze, p. 52

Sreenivasan, S.R.: 1973, Physica 67, 323, 330

Stenflo, J.0.: 1973, Solar Phys. 32, 41. 